Professional and lay opinions on multiple personality disorder

SIR: There is little knowledge of the state of professional opinion with regard to the validity of the diagnosis of multiple personality disorder. Following the publication of an article on this topic (Journal, March 1992, 160, 327-340) I took the opportunity to enclose a questionnaire with reprints which were supplied on request and some of which were also offered to colleagues. Of 90 reprints sent out, 44 were returned, of which 38 were analysable. Ten of those responding with analysable forms had received the reprint, at least in part, on my initiative.

The questions asked related to region of residence, professional identification, views on MPD, and whether the respondent wished to receive the results of the survey. Before considering the arguments in the article mentioned, 5 of the 38 responding had believed that MPD was a common condition (position (a)), 21 believed that MPD as defined in DSM-III-R occurred occasionally but was overdiagnosed (position (b)), and 12 did not believe in it (c). Subsequently, four still held it was a valid entity or condition and quite common, 15 supported the view that it occurred occasionally, and 19 rejected the diagnosis.

The present sample cannot be considered to be representative. It is potentially biased by the inclusion of colleagues whom I knew to be sympathetic to my views, although not all supported them fully and a number of others whom I might have asked to fill in forms were not approached because they had received copies of the article before the questionnaire was designed. It is also biased by the likelihood that proponents of the diagnosis, or others considering it to be a realistic diagnosis, were probably more likely to write for copies of the article than those who were not interested, or who disbelieved in it and were not attending to the literature on the topic.

The results resemble those which were obtained with a lay audience in March 1992 with the same questionnaire. At a talk given in Sarnia Ontario, at the request of the local Mental Health Association, approximately 100 questionnaires were handed out and 50 completed of which 42 were analysable. Those responding included 2 general practitioners, 1 lawyer, 1 member of the clergy, and some 35 allied health professionals, or individuals, with an interest related to their occupation, for example, social workers, psychologists, nurses, health administrators, etc., and 12 others. Before the talk 17 believed in position (a), 16 in position (b), and 9 in position (c). Subsequently, 13 held to position (a), 8 held position (b), and 21 position (c). These responses seem very like those of the largely medical audience which commented after reading the article itself. Thus informed individuals were likely either to reject or restrict the diagnosis of MPD.

From some of the comments made to me both privately and on the questionnaire forms, I have the impression that many more psychiatrists would reject the diagnosis of MPD altogether, provided that they could be satisfied that occasional fugue states with a change in temperament or apparent personality style, could still be recognised as diagnosable dissociative conditions. I think there should be no difficulty in doing that even if the diagnosis of MPD were to be given up completely.

The questionnaire is available from the author on request.

\author{
University of Western Ontario \\ London Psychiatric Hospital \\ 850 Highbury Avenue \\ PO Box 2532 \\ London \\ Ontario N6A 4 HI \\ Canada
}

HAROLD MERSKEY

\section{Cannabis and the PSE}

SIR: Dr Mathers and Professor Ghodse recently reported on a study of the psychopathology of psychotic patients admitted to hospital with positive urine tests for cannabis, compared with psychotic drug-free controls (Journal, November 1992, 161, 648-653). They found that five items on the Present State Examination (PSE) administered within one week of admission were significantly more common in the cannabis-using group. These were changed perception, thought insertion, non-verbal auditory hallucinations, delusions of control, and delusions of grandiose identity. The authors suggested two reasons for this: that it may be a chance finding due to the large number of items in the PSE, or that these may be the features of acute cannabis intoxication. In their summary, they suggest that cannabis precipitates relapse in those with major psychotic disorders. Another explanation which would fit their findings which they did not discuss would be that subjects who are experiencing a psychotic relapse may increase their drug use secondary to this, either in an attempt to self-medicate or due to the disinhibiting effects of psychosis. This drug use may then modify the presentation of the underlying illness, accounting for the differences observed using the PSE. According to this hypothesis the correct diagnosis in most cases in this 